\title{
Acalculous Cholecystitis in a Child in the Course of Salmonella Gastroenteritis. Clinical Case with a Review of the Literature
}

\author{
Velev $\mathbf{V}^{1}$, Tolekova $\mathrm{N}^{2}$, Naidenova $\mathrm{N}^{1}$, Stoilov $\mathrm{S}^{2}$, Nansenova $\mathbf{V}^{2}$ and Sopotenski $\mathbf{S}^{2}$ \\ ${ }^{1}$ Hospital for Infectious and Parasitic Diseases, Children's Clinic, Medical University of Sofia, Bulgaria \\ ${ }^{2}$ University Emergency Hospital "Pirogov", Child Surgical Clinic, Medical University of Sofia, Bulgaria
}

${ }^{*}$ Corresponding author: Valeri Velev, Chair of Infectious Diseases, Parasitology and Tropical Medicine, Medical University of Sofia, Bulgaria, Tel: 0889563412; E-mail:velev_md@abv.bg

Received date: January 3, 2018; Accepted date: January 17, 2018; Published date: January 19, 2018

Citation: Velev V, Tolekova N, Naidenova N, Stoilov S, Nansenova V, et al. (2018) Acalculous cholecystitis in a child in the course of salmonella gastroenteritis. Clinical case with a review of the literature. Pediatric Infect Dis Vol.3, No.1: 3.

Copyright: (C2018 Velev V, et al. This is an open-access article distributed under the terms of the Creative Commons Attribution License, which permits unrestricted use, distribution, and reproduction in any medium, provided the original author and source are credited.

\begin{abstract}
Salmonellosis is a typical bacterial zoonosis, and human illnesses are most often caused by the consumption of salmonella-contaminated eggs, meat, milk, etc. The disease is most common during the summer months.
\end{abstract}

Acute acalculous cholecystitis (AAC) is an acute inflammation of the gallbladder in the absence of gallstones and occurs in about $2 \%$ of Salmonella spp. infections.

We present a case of an 11-year-old boy with AAC in the course of bacterial intestinal infection with $S$. enteritidis-the development of the characteristic clinical picture, the diagnostic methods and the therapeutical behaviour.

\section{Keywords: AAC; Salmonella; Gallbladder}

\section{Introduction}

Enteropathogenic bacteria are the cause of acute infectious diarrhea, which is a global health problem. Every year, nearly 1.7 billion cases of patients with diarrhea syndrome are recorded, and diarrhea continues to be the leading cause of death among children up to 5 years worldwide, along with pneumonia. The leading causative agents of bacterial intestinal infections in Europe continue to be Campylobacter and Salmonella [1]. In Bulgaria, Salmonellosis gastroenteritis is still leading in infectious intestinal pathology, although in recent years the role of Campylobacter spp. to increase, but it is rather because of the improvement of diagnostic techniques in our microbiological network [2]. For Bulgaria in 2016, 718 cases of Salmonellosis gastroenteritis have been diagnosed, with the shingles (291) and the campylobacter (202) remaining behind them (Ministry of Health, Bulgaria, 2017).

The purpose of this report is to present the clinical manifestations and diagnostic procedures in AAC to guide clinicians and allow the rapid initiation of specific treatment.

\section{Review of Literature}

Salmonellosis is a typical bacterial zoonosis, and human illnesses are most often caused by the consumption of salmonella-contaminated eggs, meat, milk, etc. The disease is most common during the summer months when the temperature intervals allow for maximum propagation of salmonella bacteria in foods not adequately heat treated. It may occur as asymptomatic carriers, food toxic infections with abundant vomiting, or most often as gastroenteritis with multiple diarrheal stools, mixed with mucus and less frequently with blood, high fever. Severe extra-complications such as sepsis, abscesses in the abdominal and extra-abdominal organs and others [3-5] have also been reported.

Acute acalculous cholecystitis (AAC) is an acute inflammation of the gall-bladder in the absence of gallstones. AAC covers $2-15 \%$ of all cases of acute cholecystitis and occurs in about $2 \%$ of Salmonella spp. infections. Causes could be S. typhi and S. paratyphi, which is rare in Europe or more commonly $S$. enteritidis or groups B and D. It is assumed that AAC is more common in elderly men when agents are $S$. typhi and $S$. paratyphi, but in Europe and North America these salmonella species are rare [6-8]. In all cases, AAC may be progressing relatively quickly for about $48 \mathrm{~h}$ to gangrene, perforation and abscess. It is especially fast in patients over 50 years of age because of the supposed visceral atherosclerosis, which facilitates the ischemisation of gallbladder vessels $[9,10]$.

The AAC pathogenesis has not been fully elucidated, and it is currently thought to be the combination of several factors-for example, patients with metabolic syndrome and visceral atherosclerosis, besides being more prone to biliary vessels obliteration, have also had disturbances in mucosal resistance of the bladder, which predisposes to infection. It has been shown that the bacterium has tropism for the epithelium of the bile wall, localizing there and multiplying and promoting infiltration with neutrophils and production of inflammatory cytokines that alter bile stasis. Risks are also previous abdominal operations, trauma, systemic alcohol abuse $[6,9,11]$. None of these factors, however, was available to our patient. 
AAC due to primary bacterial infection in children is rare. Several cases have been reported, mainly with $S$. typhi and paratyphi as causative agents. More frequent AAC is reported in secondary extra-complications that occur days, even weeks after discontinuation of diarrhea [4-6]. Diagnosis in such cases is most often based on clinical data, ultrasound examination, and possibly computer tomography. Salmonella spp. may be absent from haemoculture but persist in bile and faeces. Most cases described in the literature are with a bad progress, complicated with gangrene of the gall bladder and often followed by perforation [3,8]. Especially often this occurs among children in Africa, Latin America and the Indian subcontinent. On the one hand, due to low hygienic standards, there are common infections with S. typhi and S. paratyphi; on the other hand, the fact that the regions are endemic for malaria, hampered the differential diagnosis at the onset of febrile seizures and often slowed down the onset of antibiotic treatment [7-9]. K. Gnassingbé et al. reported six such cases in children for a fiveyear period in Togo, West Africa, Children's Hospital [7]. In three cases in faecal samples and haemocultures were not isolated bacteria, probably due to the fact that microbiological samples are not made in the hospital during the weekend and festive days. Diagnosis is performed clinically and with an ultrasound, as well as by positive serology on Widal and Felix agglutination. In the cases of the other three children was isolated S. typhi from faecal samples. All six children had complicated cholecystitis with gangrene and cholecystectomy was required except the antibiotic treatment [7].

The mortality and morbidity rates associated with acalculous cholecystitis can be high; the illness is frequently observed in patients with sepsis or other serious conditions. The reported mortality range is $10-50 \%$ for acalculous cholecystitis as compared to $1 \%$ for calculous cholecystitis $[3,8,11]$.

\section{Clinical Case}

A boy aged 11 years is acutely ill with fever, vomiting and multiple watery stools without mucus and blood. He was treated two days at home symptomatically, but after a temporary improvement the diarrhea syndrome recovered, abdominal pains resembling colic appeared. After examination, the child was hospitalized at the University Hospital for Infectious and Parasitic Diseases, Sofia in a moderately impaired general condition, intoxicated, with signs of dehydration. The belly is soft but painful in palpation in the ileocecal region, with a lot of vivid intestinal peristalsis. From the laboratory tests there was evidence of an inflammatory process-leukocytes $15 \times 10^{9} / \mathrm{L}$ and $50 \mathrm{~mm}$ ESE.

Faeces probes were taken for microbiological, virological and parasitological studies. Treatment with infusion of glucose-salt solutions and antiemetics was started. On the second day of hospitalization, the child reported a growing abdominal pain, vomiting persisted. The belly admitted deep palpation, but it was very painful in right hypochondrium and right midline.

The control blood count showed increased leukocytosis- $27 \times$ $10^{9} / \mathrm{L}$. The child was consulted with a child surgeon and was admitted to the "Pirogov" University Hospital of Emergency
Medicine, Sofia with suspicion of acute cholecystitis. The abdominal ultrasound showed an enlarged and folded gall bladder with a thickened, flattened wall and biliary sludge in the lumen; with a small amount of SDR (Figure 1).

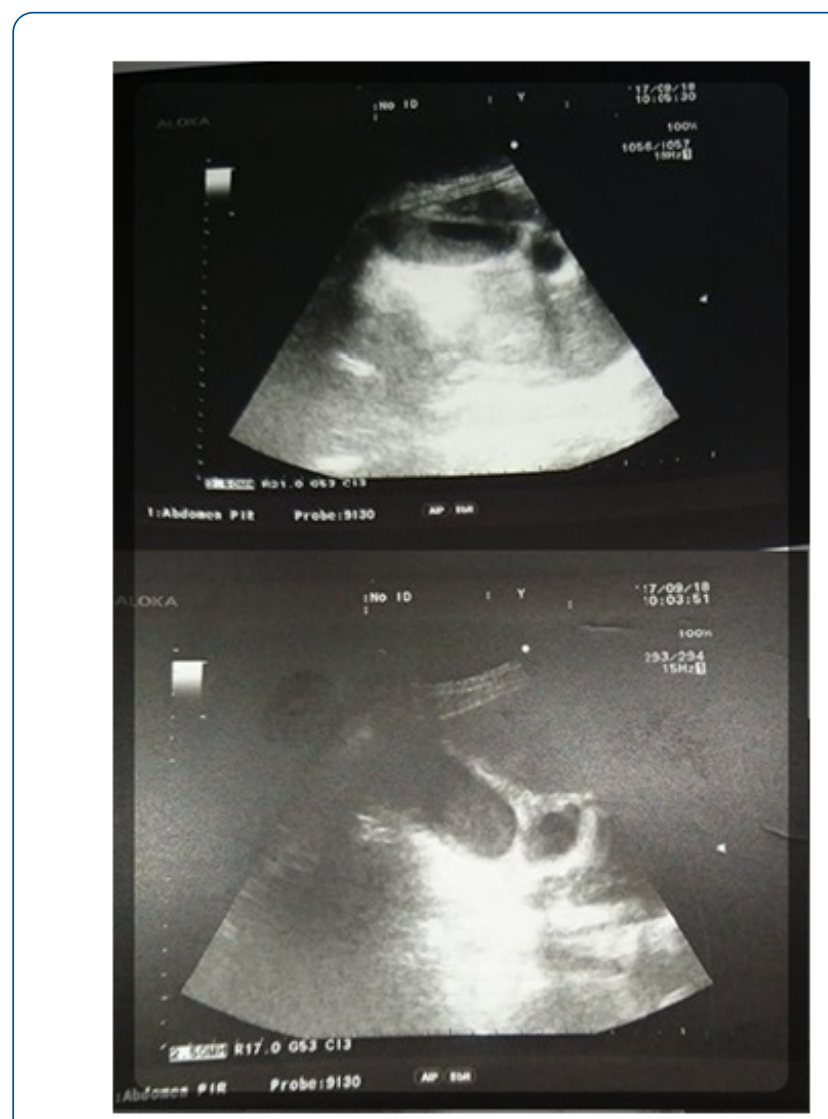

Figure 1: Abdominal ultrasonography-enlarged gall bladder, with thickened wall and bile in the lumen.

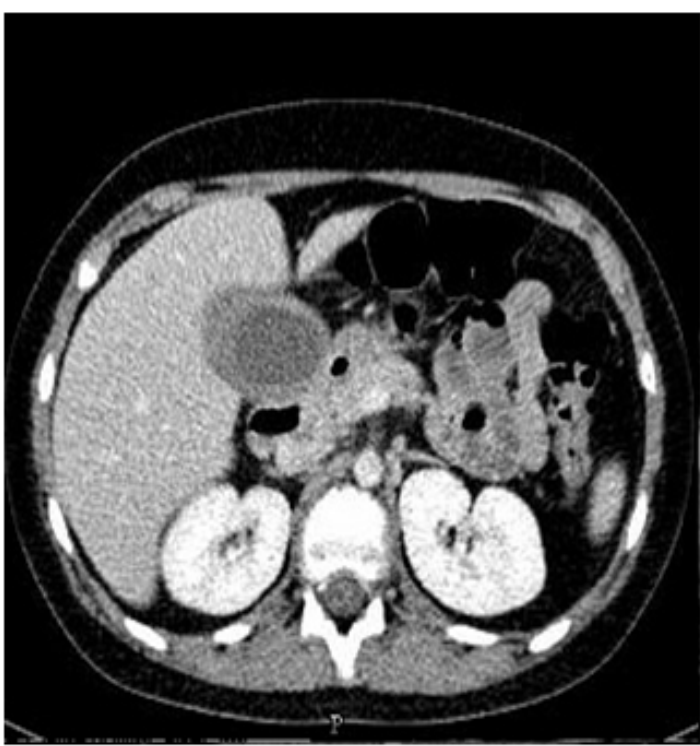

Figure 2: Small pelvis-enlarged gallbladder with thickened wall and paravesical fluid. 
From CAT of small pelvis-enlarged gallbladder $120 / 40 \mathrm{~mm}$ thickened wall and paravesical fluid, also fluid found and in the small pelvis. Enlarged mesenteric lymph nodes in lower right quadrant. The appendix was intact (Figure 2).

A seizure of Salmonella enteritidis was reported at the 72 hour of the faeces probe examination. The changes were interpreted as acalculous cholecystitis with periholecystitis in the course of salmonella gastroenteritis. Due to lack of complications, conservative intravenous treatment with Amikacin and Metronidazole was performed. The patient recovered completely after the antibiotic treatment.

\section{Discussion and Conclusion}

The main cause of acalculous cholecystitis is gallbladder stasis with resulting stagnant bile. This is observed most commonly in patients with sepsis, patients in intensive care units, patients on long-term total parenteral nutrition, those with cardiovascular disease, patients with diabetes. In children without chronic illness, it is mostly about a complication of salmonellosis, with a favorable course with at early conservative treatment $[3,6,11]$.

Although rarely a complication of Salmonellosis, AAC, once occurred, it can rapidly progress to a severe, life-threatening condition. In this case, early diagnosis, mainly imaging, led to a positive outcome without the need for surgical treatment. In abscessing, gangrene or other complications, cholecystectomy, open or laparoscopic in the course of AAC, has no alternative. Etiological antibiotic therapy should always include "Metronidazol", not only against a possible anaerobic intestinal flora but also against probable conditional pathogens or pathogenic protozoa in the course of dysbacteriosis [5].

Any abdominal pain in the course of acute intestinal infection with high leukocytosis and fever should be suspected of having a severe surgical abdomen, in particular appendicitis or AAS.

\section{References}

1. Sala Farré MR, Osorio SD, Arias VC, Simó SM, Recasens Ra, et al. (2015) Campylobacter and Salmonella acute gastroenteritis: epidemiology and health care utilization. Med Clin (Barc) 145: 294-297.

2. Pavlova M, Elina GD, Katucha II, Galina DA, Ivan NI, et al. (2016) Multiplex PCR assay for identifi cation and differentiation of Campylobacter jejuni and Campylobacter coli isolates. Folia Medica 58 : 95-100.

3. Canut Blasco A, Brezmes Valdivieso MF, Antolín Ayala MI, Yagüe Muñoz A (1992) Focal infections caused by non-typhi Salmonella: A review of our case series and comparison with other series. Rev Clin Esp 191: 71-5.

4. Lalitha MK, John $R$ (1994) Unusual manifestations of salmonellosis-a-surgical problem. Q J Med 87: 301-309.

5. Velev V, Durveniashka N, Naidenova N, Ilchova V, Tomova I, et al. (2014) Campylobacteriosis and salmonellosis-differential diagnostic study in children. Bul Med J 8: 24-28.

6. Ganpathi IS, Diddapur RK, Eugene H, Karim M (2007) Acute acalculouscholecystitis: Challenging the myths. HPB (Oxford) 9: 131-134.

7. Gnassingbé K, Katakoa G, Kanassoua KK, Adabra K, Mama Wa, et al. (2013) Acute cholecystitis from typhic origin in children. Afr J Paediatr Surg 10: 108-111.

8. Shimoni Z, Pitlik S, Leibovici L, Samra Z (1999) Nonthyphoid salmonella bacteraemia: Age-related differences in clinical presentation, bacteriology, and outcome. Clin Infect Dis 28: 822-827.

9. Benjelloun EB, Chbani L, Toughrai I, Ousadden A, Mazaz K, et al. (2013) A case report of acute acalculous cholecystitis due to Salmonella paratyphi B complicated by biliary peritonitis. Pan Afr Med J 16: 127.

10. Menendez A, Arena ET, Guttman JA, Thorson L, Vallance Ba, et al. (2009) Salmonella infection of gallbladder epithelial cells drives local inflammation and injury in a model of acute typhoid fever. J Infect Dis 200: 1703-1713.

11. Wang AJ, Wang TE, Lin CC, Lin SC, Shih SC (2003) Clinical predictors of severe gallbladder complications in acute acalculous cholecystitis. World J Gastroenterol 9: 2821-2823. 